\title{
Efficient mutation screening for cervical cancers from circulating tumor DNA in blood
}

Sun-Young Lee ${ }^{1,2 \dagger}$, Dong-Kyu Chae ${ }^{3 \dagger}$, Sung-Hun Lee ${ }^{3}$, Yohan Lim³ ${ }^{3}$ Jahyun An ${ }^{3}$, Chang Hoon Chae ${ }^{4}$, Byung Chul Kim ${ }^{3}$, Jong Bhak ${ }^{3,5,6}$, Dan Bolser ${ }^{6}$ and Dong-Hyu Cho ${ }^{2,7^{*}}$ (D)

\begin{abstract}
Background: Early diagnosis and continuous monitoring are necessary for an efficient management of cervical cancers (CC). Liquid biopsy, such as detecting circulating tumor DNA (ctDNA) from blood, is a simple, non-invasive method for testing and monitoring cancer markers. However, tumor-specific alterations in ctDNA have not been extensively investigated or compared to other circulating biomarkers in the diagnosis and monitoring of the CC. Therfore, Next-generation sequencing (NGS) analysis with blood samples can be a new approach for highly accurate diagnosis and monitoring of the CC.
\end{abstract}

Method: Using a bioinformatics approach, we designed a panel of 24 genes associated with CC to detect and characterize patterns of somatic single-nucleotide variations, indels, and copy number variations. Our NGS CC panel covers most of the genes in The Cancer Genome Atlas (TCGA) as well as additional cancer driver and tumor suppressor genes. We profiled the variants in ctDNA from 24 CC patients who were being treated with systemic chemotherapy and local radiotherapy at the Jeonbuk National University Hospital, Korea.

Result: Eighteen out of 24 genes in our NGS CC panel had mutations across the 24 CC patients, including somatic alterations of mutated genes (ZFHX3-83\%, KMT2C-79\%, KMT2D-79\%, NSD1-67\%, ATM-38\% and RNF213-27\%). We demonstrated that the RNF213 mutation could be used potentially used as a monitoring marker for response to chemo- and radiotherapy.

Conclusion: We developed our NGS CC panel and demostrated that our NGS panel can be useful for the diagnosis and monitoring of the $\mathrm{CC}$, since the panel detected the common somatic variations in CC patients and we observed how these genetic variations change according to the treatment pattern of the patient.

Keywords: Cervical cancer, Next-generation-sequencing, Circulating tumor DNA, Cancer panel, Genomic alteration

\footnotetext{
* Correspondence: obgyn2001@jbnu.ac.kr

†Sun-Young Lee and Dong-Kyu Chae contributed equally to this work.

${ }^{2}$ Research Institute of Clinical Medicine of Jeonbuk National

University-Biomedical Research Institute of Jeonbuk National University Hospital, Jeonju, Republic of Korea

7Department of Obstetrics and Gynecology, Jeonbuk National University

Hospital-Jeonbuk National University Medical School, Jeonju, Jeonbuk, Republic of Korea

Full list of author information is available at the end of the article
}

C C The Author(s). 2020 Open Access This article is licensed under a Creative Commons Attribution 4.0 International License, which permits use, sharing, adaptation, distribution and reproduction in any medium or format, as long as you give appropriate credit to the original author(s) and the source, provide a link to the Creative Commons licence, and indicate if changes were made. The images or other third party material in this article are included in the article's Creative Commons licence, unless indicated otherwise in a credit line to the material. If material is not included in the article's Creative Commons licence and your intended use is not permitted by statutory regulation or exceeds the permitted use, you will need to obtain permission directly from the copyright holder. To view a copy of this licence, visit http://creativecommons.org/licenses/by/4.0/ The Creative Commons Public Domain Dedication waiver (http://creativecommons.org/publicdomain/zero/1.0/) applies to the data made available in this article, unless otherwise stated in a credit line to the data. 


\section{Background}

Cervical cancer (CC) is the third most frequently diagnosed cancer and the fourth most common cause of cancer-related death among women worldwide, particularly in developing countries [1]. Although the development of a screening method for human papillomavirus (HPV)-based diagnosis for $\mathrm{CC}$ and $\mathrm{HPV}$ vaccination have lowered the incidence and death rate, this cancer still remains among the most common causes of cancer-related death in women [2]. High-risk human papillomavirus (HR-HPV), which is difficult to eradicate by the host immune system, infects the epithelial layer of the cutaneous and mucosal surfaces $[3,4]$. The mechanism of HR-HPV infection is an important carcinogenic factor that increases the risk of CC development over time. It has been reported that $15-30 \%$ of patients with earlystage CC experience recurrences after surgical operation, and half of those who previously had recurrent cancer show a higher risk of another recurrent cancer within 3 years after primary treatment. Thus, it is recommended that patients visit clinics for checkups every 3-4 months for the first 2 years, and every 6-12 months for the next 3-5 years after initial treatment to monitor the recurrence of CC [5]. During a check-up for cancer recurrence, cervical cytology, measurement of squamous cell carcinoma antigen and CA-125 in the blood, and medical imaging techniques such as computed tomography, magnetic resonance imaging, and positron emission spectroscopy are performed. However, the cervical cytology and the blood tests are limited by their low sensitivity and specificity. Medical imaging techniques can be employed to improve the detection of cancer recurrence, but they are costly and involve radiation exposure. Recent liquid biopsy studies showed that cell-free DNA (cfDNA), which originates from the apoptosis and necrosis of normal and tumor cells may be valuable for monitoring tumor behaviors and treatment responses [6-8]. The detection of HPV16 and HPV18 DNA or alterations in the cfDNA of patients with CC patients is used as biomarkers for recurrence monitoring [9-11].

For these reasons, we built a custom NGS panel consisting of 24 genes related to gynecological cancers. In this study, we assessed the clinical utility of analyzing gene mutations in $\mathrm{CC}$ patients who have a medical history of chemotherapy and radiotherapy, profiling the genetic variations in cfDNA from 24 patients. As a result, we were able to obtain the mutational variations in ctDNA and observe their patterns over time, which can be used to detect the phases of $\mathrm{CC}$, monitor the tumor status, and predict therapeutic responses.

\section{Methods}

\section{Samples and clinical data}

A total of 24 CC patients were enrolled in a prospective cohort at the Jeonbuk National University Hospital. All subjects provided an informed consent to participate in the study, and all clinical specimens were collected with approval from the institutional review board (IRB No. CUH2017-04-018-001) and ethics committee of Jeonbuk National University Hospital. Total of $7 \mathrm{ml}$ of whole peripheral blood was collected into EDTA tubes from each patient approximately 1 week before chemotherapy. Genetic variations were analyzed using our NGS CC panel. For each patient, mutations were characterized from both cfDNA, plasma, and peripheral blood mononuclear cells (PBMCs).

\section{Sample preparation}

PBMCs were isolated from $7 \mathrm{ml}$ of whole blood by density gradient centrifugation in Ficoll-Paque ${ }^{\mathrm{Tw}}$ PLUS (GE Healthcare, Little Chalfont, UK). We extracted cfDNA from isolated plasma using the QIAamp Circulating Nucleic Acid Kit (Qiagen, Hilden, Germany) according to the manufacturer's instructions. The extracted DNA was quantified using a Qubit 3.0 fluorometer (Invitrogen, Carlsbad, CA, USA). We analyzed the quality of cfDNA using the 2100 Bioanalyzer (Agilent Technologies, Santa Clara, CA, USA) to detect genomic DNA contamination.

\section{Next-generation-sequencing}

A total of 2265 amplicons were designed in two primer pools to capture the targeted regions. Amplicon size was designed to be 125-140 base pairs (bp), and the total number of bases covered by the amplicons was 169.34 kb. A total of $10 \mathrm{ng}$ of cfDNA and PBMC-derived DNA was used for library construction. Library preparation was performed using an Ion Ampliseq Library Kit 2.0 (Thermo Fisher Scientific, Waltham, MA, USA) according to the manufacturer's instructions. We used the Ion Express Barcode Adaptors Kit (Thermo Fisher Scientific) for sample multiplexing, and libraries were purified using the Agencourt AMPure XP reagent (Beckman Coulter, Brea, CA, USA). Libraries were quantified using the Qubit 3.0 fluorometer and 2100 Bioanalyzer. Template preparation of the libraries was performed using the Ion Chef Instrument (Thermo Fisher Scientific) with an Ion 540 Chef Kit (Thermo Fisher Scientific). Multiplexed templates were subjected to sequencing on the Ion S5 XL system (Thermo Fisher Scientific). PBMCs were evaluated to analyze somatic mutations and exclude germline mutations. Our panel can detect $0.1 \%$ tumor mutated cfDNA to normal cfDNA (range of read depths from 1000x to 3902x with a median read depth of 1554x). However, given the percentage of mutated 
tumor present in cfDNA, we set cut-off value of variation as $1 \%$.

\section{Variant analysis}

The human genome sequence hg19 was used as a reference for variant calling. Sequence and data analyses were performed using Torrent Suite software (5.8.0). Sequencing coverage analysis was performed using coverage Analysis (5.8.0.1) plugins, and VCF files were generated using the variantCaller (5.8.0.19) plugins. Annotations of the variants were obtained using Ion Reporter (5.10.2.0) software. To filter out the potential sequencing background noise, we excluded common Korean singlenucleotide variations, which are from KoVariome (http://variome.net). whole genome sequence database of 50 healthy unrelated Korean individuals $[12,13]$ and patient specific normal variants detected in PMBCs. After filtering (described above), the resulting cfDNA somatic mutations were annotated using the COSMIC database (https://cancer.sanger.ac.uk/cosmic) for comparison with previously reported variants.

\section{Results}

\section{CC targeted NGS panel}

Generally, liquid biopsies accompanying genomic analysis alone cannot identify all the features of the primary tumor. However, genetic alterations occurring in cancer patients must reflect cancer type specific mutations. Using our CC-targeted NGS panel, we first tried to detect any $\mathrm{CC}$ specific genetic variation in the patients. Then we sought to check the general mutation patterns of usual oncogenes, such as PIK3CA or TP53.

Based on TCGA database, we designed NGS CC-panel (Table 1) consists of 24 genes that are known to occur in gynecologic cancer at a high frequency. It contains $67 \%$ of genes that have been previously reported as significantly mutated in many cancers (SMGs - PIK3CA, EP300, FBXW7, HLA-B, PTEN, NFE2L2, ARID1A, KRAS, and MAPK [14]). This panel also covers $55 \%$ of the top 20 , and $80 \%$ of the top ten genes detected in CC-related tumor tissue, according to the COSMIC data. The included genes are PIK3CA, KRAS, TP53, PTEN, KMT2C, FBXW7, KMT2D, EP300, ARID1A, FAT1, and ZFHX3. The other genes in our panel are related to tumor suppressor activity [15-17].

Table 1 Gene list in customized CC panel

\begin{tabular}{lllll}
\hline ARID1A & ATM & BCOR & CHD4 & CTCF \\
CTNNB1 & EP300 & FAT1 & FAT4 & FBXW7 \\
FGFR2 & KMT2C & KMT2D & KRAS & NSD1 \\
OR14K1 & PIK3CA & PIK3R1 & POLE & PTEN \\
RNF213 & TP53 & TRRAP & ZFHX3 & \\
\hline
\end{tabular}

\section{Evaluation of panel through reference materials}

We verified the NGS panel with standard materials to determine its sensitivity. The standard material (Horizon Discovery, Cambridge, UK) contains mutations in PIK3$C A(\mathrm{E} 545 \mathrm{~K})$ and $K R A S(\mathrm{G} 12 \mathrm{D})$ genes. For accuracy, the sequencing was performed under the same conditions as the patient samples (10 ng input giving 1000-fold coverage). As a result of using 5\% Multiplex I cfDNA Reference Standard, 6.3\% variation was detected. Subsequently, 1.3 and $0.13 \%$ of allele frequency were identified under the utilization of 1 and $0.1 \%$ of standard material (Fig. 1). In addition, the average variations for PIKC $3 A$ and KRAS were 1.33 and $1.6 \%$, respectively, based on the verification using $1 \%$ standard material. The allele frequencies of PIK3CA and KRAS were 0.27 and $0.4 \%$, respectively. All samples were evaluated in triplicate, and the detection of standards confirmed that our panel was sensitive enough to detect $0.1 \%$ of genetic variation. To verify the sensitivity and specificity of this panel, the gene mutations were identified by digital droplet PCR, which indicated that the sensitivity for the PIK3CA gene was $88.9 \%$ and specificity was $100 \%$. The result of the ddPCR also confirmed $100 \%$ sensitivity and specificity in the detection of the KRAS gene (Table 2).

\section{Characteristics of patients}

Twenty-four patients with $\mathrm{CC}$ were enrolled in this study. The criteria for enrollment was not casecontrolled; therefore, patients were not specifically classified by cancer stage or histology, which could have introduced bias. The clinical and histopathological characteristics of these patients are summarized in Table 3. Blood samples were collected from the patients approximately 1 week prior to primary treatment. Genetic alteration was monitored in four patients who agreed to provide blood during the treatment, and their blood samples were drawn three times for the prognosis prediction. The median age in our cohort was 61 years and $25 \%(n=6)$ of the patients had disease at stage I, followed by stage II $(n=11,46 \%)$, stage III $(n=3,13 \%)$, and stage IV $(n=4,17 \%)$ disease. Histology analysis revealed that cases varied from adenocarcinoma to invasive $\mathrm{CC}$, and squamous cell carcinoma was the most common (79\%). The stages of CC were diagnosed using imaging-based methods (computed tomography and magnetic resonance imaging). Most of the patients were treated with cisplatin-based chemotherapy and radiation therapy, and the patients with small cell neuroendocrine carcinoma were treated with combination of cisplatin, paclitaxel, and bevacizumab. The radiation therapy regime was mainly administered to the pelvic site with $54 \mathrm{~Gy} / 30 \mathrm{fx}$ followed by ICR (Intracavity radation) $24 \mathrm{~Gy} / 6 \mathrm{fx}$. 


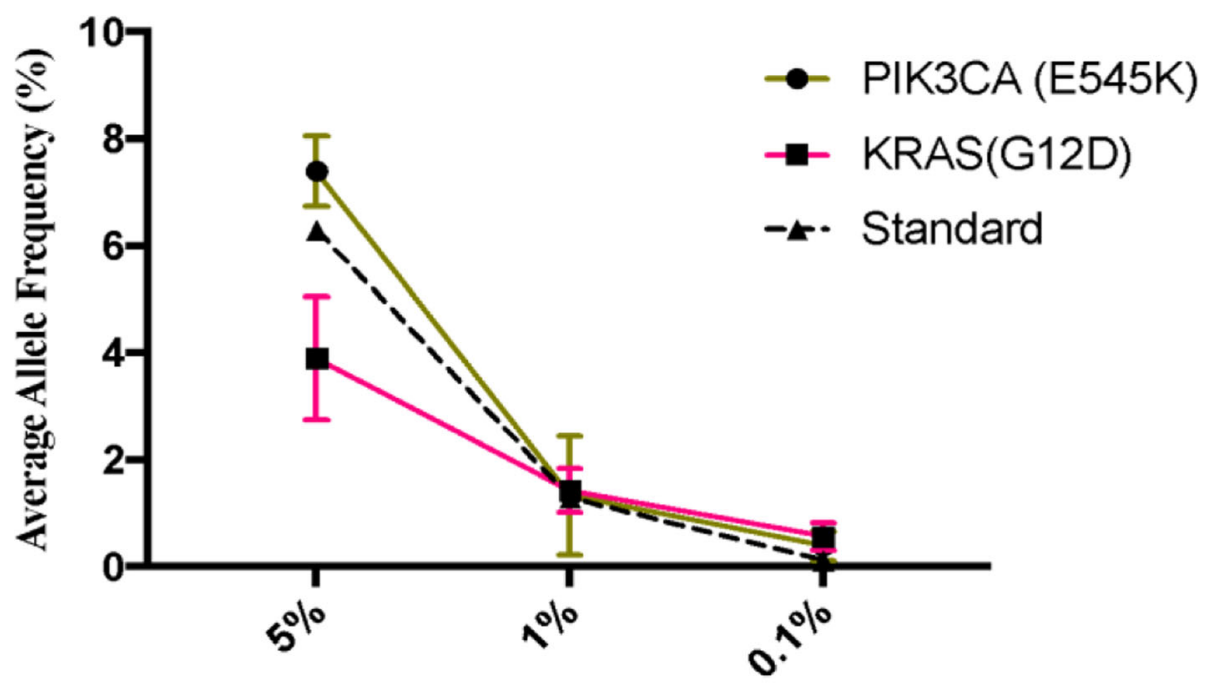

Expected Allele Frequency

Fig. 1 Verification with standard material. Based on comparisons using standard substances, NGS analysis confirmed the allele frequency of PIK3CA(E545K) and KRAS(G12D) with 1\% of accuracy

\section{Genomic alterations in CC patients}

The initial study was conducted by screening for overall genetic variation in patients with CC using our NGS panel. To explore the profiles of molecular variants, we analyzed cfDNA and PBMC that were extracted from the blood of 24 CC patients.

Twenty-four CC patients were sorted by different cancer stages and histology features (Fig. 2a and Supplemenatary Table 1). All patients with stages III and IV had the homogenous histology type as squamous cell carcinoma. Three patients (among the six patients with stage I disease) showed the same histology. Among the 24 genes in the list, alterations were found in 18 genes (75\%) and no mutations were found in the remaining six genes (BCOR, CTNNB1, FGFR2, OR14K1, POLE, and KRAS). Genetic mutations in ZFHX3, KMT2C/D, and NDS1 were detected in 20 (83\%), 19 (79\%), and $16(67 \%)$

Table 2 Verification of NGS panel using ddPCR as a gold standard

\begin{tabular}{|c|c|c|c|c|c|}
\hline & Positive & Negative & Total & Sensitivity & Specificity \\
\hline \multicolumn{6}{|c|}{ PIK3CA(E545K) } \\
\hline Positive & 8 & 1 & 9 & $88.9 \%$ & $100 \%$ \\
\hline Negative & 0 & 3 & 3 & & \\
\hline Total & 8 & 4 & 12 & & \\
\hline \multicolumn{6}{|l|}{ KRAS(G12D) } \\
\hline Positive & 9 & 0 & 9 & $100 \%$ & $100 \%$ \\
\hline Negative & 0 & 3 & 3 & & \\
\hline Total & 9 & 3 & 12 & & \\
\hline
\end{tabular}

women with CC, respectively (Fig. 2b). These genes have been reported as tumor suppressors and are prevalent in other cancer-related diseases [18-23]. According to the data published by TCGA, PIK3CA (26\%), EP300 (11\%), FXBW7 (11\%), and PTEN (8\%) are the common genetic variants in CC [14]. However, our analysis showed that alterations within these genes occurred in 12.5, 12.5, 4, and $8 \%$ of the cases, respectively. Out of all the variant types, the missense mutations (24\%) accounted for the largest number of variant types. Mutation patterns with two or more mutation types (such as missense and frameshift) were found in 15 patients. Frameshift insertions and deletions were found in only five patients. Overall, at least three genetic variants were found in all patients, with an average of 9 mutations per patient

Table 3 Patient characteristics

\begin{tabular}{ll}
\hline Entire cohort & 24 \\
\hline Histology & $19(79 \%)$ \\
Squamous cell carcinoma & $2(8 \%)$ \\
Endocervical adenocarcinoma & $1(4 \%)$ \\
Small cell neuroendocrine carcinoma & $1(4 \%)$ \\
Low-grade squamous intraepithelial neoplasia & $1(4 \%)$ \\
Invasive cervical cancer & \\
Pathogenic stage & $6(25 \%)$ \\
Stage I & $11(46 \%)$ \\
Stage II & $3(13 \%)$ \\
Stage III & $4(17 \%)$ \\
Stage IV &
\end{tabular}



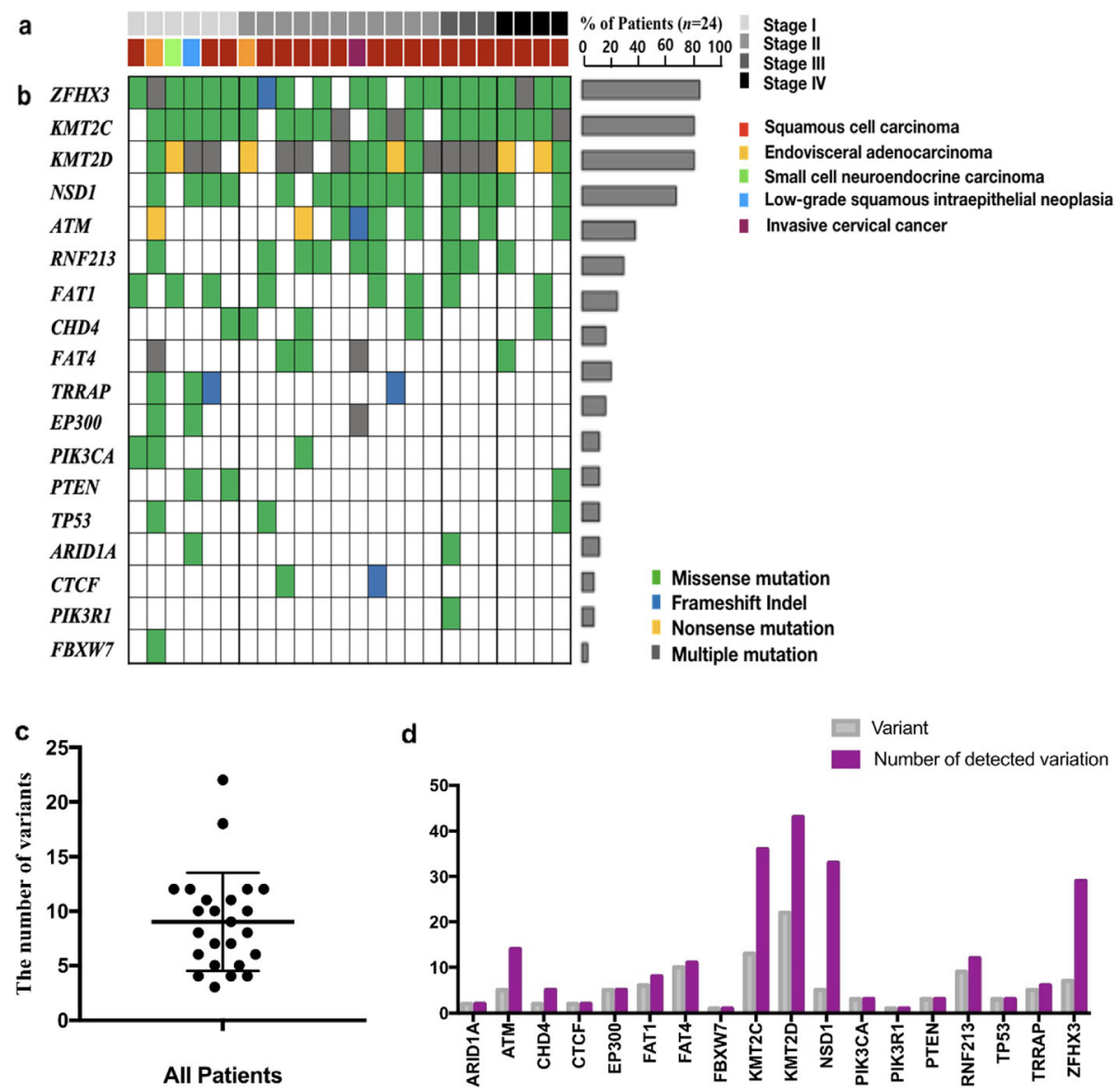

Fig. 2 Somatic alterations in CC. a. Stages and the type of CC histology are represented. $\mathbf{b}$. The Genetic variation panel lists the patient-specific variations, as well as the sequence of these variations. c. Dot plot indicates the number of variants in CC patients. d. Gray bar graphs show information for all gene variants, and purple bar graphs show the number of amplicons of gene variants detected in all patients

(Fig. 2c). The largest number of mutations was 22 variants in one patient. The total number of distinct mutations was 217 across all patients. After analyzing all variants of each gene, the most commonly detected variations were located in KMT2D [23], followed by the KMT2C [13], FAT4 [10], RNF213 [9], and $Z F H X 3$ [7] variants (Fig. 2d). Most mutations in cancer suppressor genes were evenly detected across all stages of cancer, whereas cancer driver gene variants were found mainly in the early stages of cancer (stage I and II).

\section{Variant allele frequency for patient monitoring}

Among the 24 patients, 4 patients who agreed to monitor were selected. All assigned patients had been diagnosed with $\mathrm{CC}$ and showed the same general squamous cell carcinoma histology. The chemotherapy regimens were CDDP \#6 (cisplatin) and radiotherapy was operated on pelvis with $54 \mathrm{~Gy} / 30 \mathrm{fx}$ followed by ICR $24 \mathrm{~Gy} / 6 \mathrm{fx}$.

Patient 1 was 74 years old and was confirmed to have squamous cell carcinoma (stage IV) by surgical pathological examination. The follow-up period was approximately 19 months. The CC panel analysis revealed a total of four gene mutations (Fig. 3). In addition to KMT2C and ZFHX3 mutations found in most patients, PIK3CA and RNF213 mutations were also detected, and RNF213 mutations changed over 18 months of the examination. Initial test findings revealed that the uterine cervix had an intense increment in mass and was approximately $5 \mathrm{~cm}$ in size stained with fluorodeoxyglucose (FDG) and may have been invaded into the bladder posterior wall. The patient was treated with CDDP for approximately 2.5 months. After undergoing chemotherapy (P2), a therapeutic effect was confirmed (partial response; PR). The KMT2C and PIKC3CA mutations, which were elevated in number in the early stages of chemotherapy, declined over time. By the time of the third examination, no PIK3CA and RNF213 mutations were detected. The score for the FATHMM (http://fathmm.biocompute.org.uk) pathological prediction is 0.98 for $K M T 2 C$ and 0.96 for PIK3CA, respectively. The third examination showed no residues from previous tumor observation (complete response; CR). 

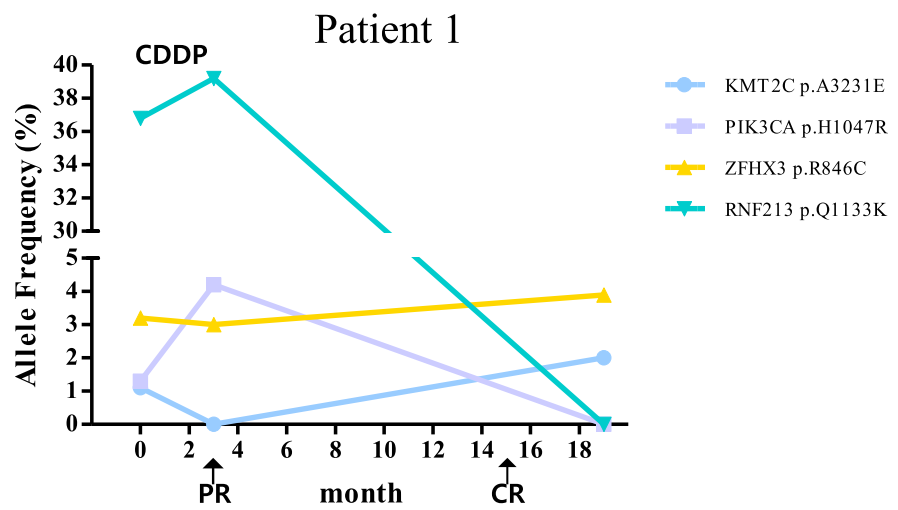

$=\quad$ RNF213 p.Q1133K

\begin{tabular}{|c|c|c|c|c|c|c|l|}
\hline \multicolumn{2}{|c|}{ Genes } & COSMIC & P1 & P2 & P3 & FATHMM prediction \\
\hline \multirow{2}{*}{ KMT2C } & c.943G>A & p.G315S & COSM1179668 & 03.4 & 01.3 & 4.7 & Pathogenic (score 0.98) \\
\cline { 2 - 8 } & c.967A>T & p.A3231E & COSM1488408 & 01.1 & 00.0 & 2.0 & \\
\hline PIK3CA & c.3140A>G & p.H1047R & COSM249874 & 01.3 & 04.2 & 0.0 & Pathogenic (score 0.96) \\
\hline \multirow{2}{*}{ RNF213 } & c.3397C>A & p.Q1133K & COSM148362 & 36.8 & 39.2 & 0.0 & \\
\hline ZFHX3 & c.2536C>T & p.R846C & COSM973550 & 03.2 & 03.0 & 3.9 & \\
\hline
\end{tabular}

Fig. 3 Patient specific features of tumor suppressor gene mutation. PR: partial response. CR: complete response. P1: Period 1, P2: Period 2, P3: Period 3

Patient 2 was 56 years old and was confirmed to have squamous cell carcinoma (stage II) by surgical pathological examination. The follow-up period was approximately 19 months. The patient did not undergo any further surgery, and the imaging findings revealed FDGavid malignancy in the uterine cervix with extension into the uterine body and fundus. A total of three genetic mutations were found, which tended to decrease the allele frequencies overall with initial chemotherapy (Fig. 4). The variation of allele frequency in RNF213, which did not appear in the first and second examinations, until it was found at a $3.7 \%$ AF during the third examination. The mutation of KMT2D gene, which is considered as pathologic (0.84) according to the FATHMM prediction, was detected in the first screening but disappeared in the second screening and then reappeared in the third screening. The genetic variation of ZFHX3 was found to decrease in the early phase of chemotherapy $(2.1 \%)$, but increased over time (7.2\%). At the third clinical examination, a mass distinct from the cervix was detected, and a slight thickening across the endometrium was also detected; otherwise, no measurable enlarged lymph nodes or fluid collections were observed around the lesion. As a result, although the CC size did not increase significantly, this patient was diagnosed with partial response to chemotherapy, due to other factors around cervix lesion site.
Patient 3 was 48 years old and had squamous cell carcinoma type CC (stage II) which tended to be keratinized. The patient had mutations in two genes (KMT2D and $Z F H X 3$ ); the $K M T 2 D$ mutation, which is considered as pathogenic (score 0.84), disappeared by the second and third screening (Fig. 5). The initial screening showed localized metastasis to the lymph node (LN) region of the uterine cervix and multiple myomas in the uterus. The treatment of this patient involved approximately 2 months of chemotherapy. Ten months later, the third examination was performed. Examination revealed no lesion sites in the uterine cervix. The LN of approximately $1.3 \mathrm{~cm}$ was still visible but was decreased in size. Additionally, leiomyoma of less than $4 \mathrm{~cm}$ was observed in the uterus. However, several uterine leiomyoma and endometrial polyps were observed (PR).

Patient 4 was 51 years old and had stage III cancer. The patient was followed up for approximately 13 months. The patient' positron emission spectroscopy images showed an FDG-avid mass (SUVmax $=22.33$ ) of a metabolic size of approximately $5 \times 2.5 \mathrm{~cm}$ in the uterine cervix in the abdomen and pelvis. The metabolic length was approximately $5.5 \mathrm{~cm}$, extending into the vagina and abutting the bladder base. Both external iliac LNs were up to $8 \mathrm{~mm}$ in diameter and showed FDG uptake. Other internal iliac LNs appeared to be small in size and did not show FDG uptake. In this patient, three major gene 

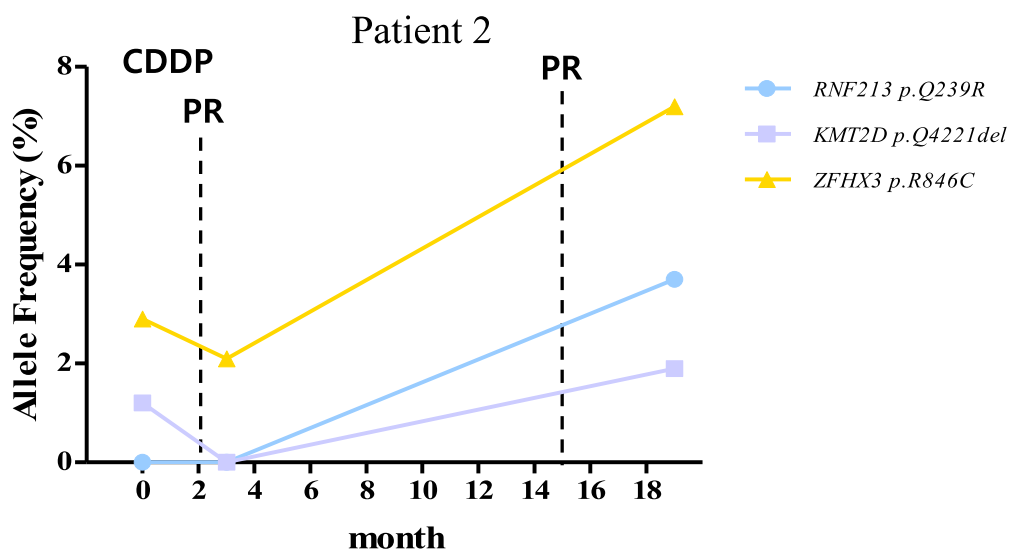

\begin{tabular}{|l|c|c|c|c|c|c|c|}
\hline \multicolumn{2}{|c|}{ Genes } & COSMIC & $\begin{array}{c}\text { P1 } \\
\mathbf{1 7 . 0 5 . 1 5}\end{array}$ & $\begin{array}{c}\text { P2 } \\
\mathbf{1 7 . 0 8 . 0 1}\end{array}$ & $\begin{array}{c}\text { P3 } \\
\mathbf{1 8 . 1 2 . 0 5}\end{array}$ & $\begin{array}{c}\text { FATHMM } \\
\text { prediction }\end{array}$ \\
\hline KMT2D & c.12662_12664delAGC & p.Q4221del & COSM940003 & 1.2 & 0.0 & 1.9 & $\begin{array}{c}\text { Pathogenic } \\
\text { (score 0.84) }\end{array}$ \\
\hline RNF213 & c.716A>G & p.Q239R & COSM3523349 & 0.0 & 0.0 & 3.7 & $\begin{array}{c}\text { Neutral (score } \\
0.05)\end{array}$ \\
\hline ZFHX3 & c.2536C>T & p.R846C & COSM973550 & 2.9 & 2.1 & 7.2 & \\
\hline
\end{tabular}

Fig. 4 Patient specific features of tumor suppressor gene mutation

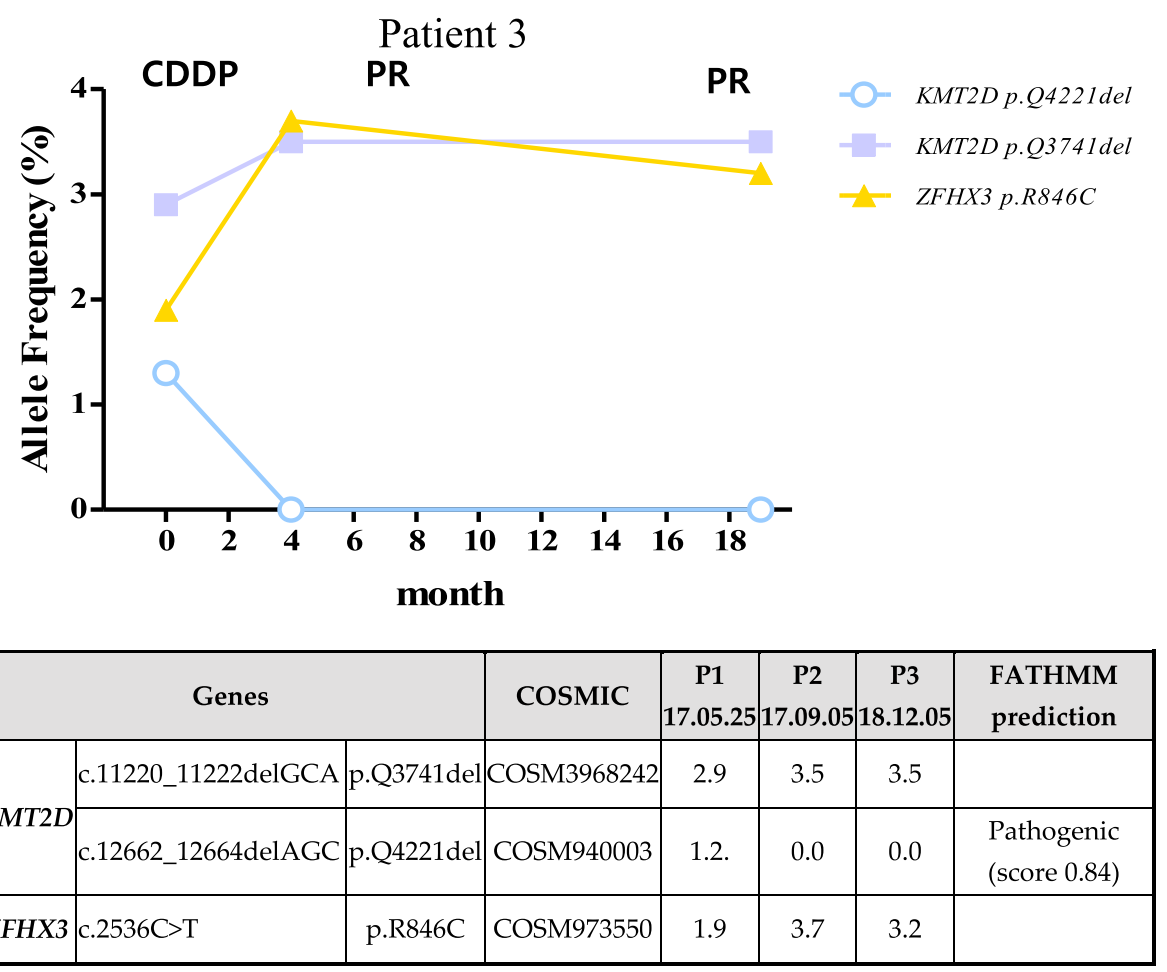

Fig. 5 Patient specific features of tumor suppressor gene mutation 
mutations (KMT2D, NSD1 and RNF213) were found (Fig. 6). After treatment, the two of the three genetic variants disappeared. The RNF213 gene mutation was not observed in the first screening, whereas its AF was found in the second and third screenings. After the third examination, lesions observed in the cervix and vagina anterior portions were not visible. There was no change in the sub centimeter-sized myomas in the uterine fundus. No significantly enlarged LN was observed in the pelvic cavity, and no abnormal fluid collection was observed. There were no abnormal findings in the metastases, urinary bladder, and rectum in the pelvic bone. In conclusion, a complete response was confirmed based on the difficulty in detecting the lesion site.

\section{Discussion}

In the present study, we analyzed cfDNA from patients with CC by NGS using a customized panel of 24 cancerrelated genes using the Ion Torrent system. The study was conducted to identify cfDNA mutations and explore their effectiveness in diagnosing and monitoring CC. There were three key challenges faced during this study. (i) All 24 subjects provided cancer-positive samples, however, we did not have non-patients samples to compare with. For a better understanding of the cancerpositive DNAs, samples from healthy donors must be included in cfDNA library preparation and sequencing, and cut-off values must also be validated more robustly for variant calling. (ii) In the analysis of assay specificity, there was excessive noise in variant calling. According to TCGA, there was an average of 4 mutations per Mbp. Although we minimized the technical errors and germline variants, there were approximately five mutations in $52 \mathrm{Mbp}$. Various data must be included to demonstrate the specificity of somatic variant calling. These data may include a list of specific variant locations and nucleotide changes across all samples. Other than established hotspot mutations, repeated variants across multiple samples may indicate technical errors. (iii), The detection sensitivity was limited. The levels of mutations in PIK3CA, KRAS, and TP53, the most relevant mutated genes in $\mathrm{CC}$, were lower than expected. Because of the small positive cohort group (24 CC-positive patients), statistical analysis was difficult. Technical evaluation must be performed to further evaluate the assay sensitivity.

Recent studies have shown that the role of $K M T 2 C / D$ gene is generally known to perform enhancer regulation by deposition of H3K4me1 in normal cells [24, 25] and a transcription regulator in cancer [26, 27]. Likewise the $K M T 2 C / D$ gene, which may play an important role in cancer, is reported to have a frequency of up to $89 \%$

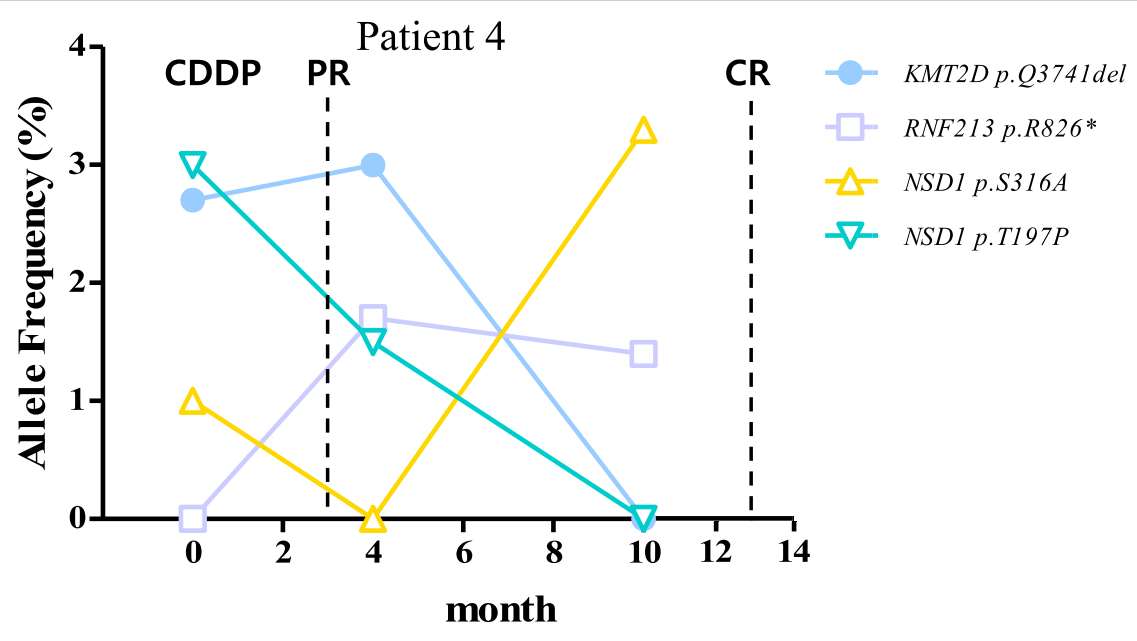

\begin{tabular}{|c|c|c|c|c|c|c|c|}
\hline \multicolumn{2}{|c|}{ Genes } & COSMIC & $\begin{array}{c}\text { P1 } \\
\mathbf{1 7 . 1 1 . 0 6}\end{array}$ & $\begin{array}{c}\text { P2 } \\
\mathbf{1 8 . 0 3 . 0 7}\end{array}$ & $\begin{array}{c}\text { P3 } \\
\mathbf{1 8 . 0 9 . 1 9}\end{array}$ & $\begin{array}{c}\text { FATHMM } \\
\text { prediction }\end{array}$ \\
\hline KMT2D & c.11220_11222delGCA & p.Q3741del & COSM3968242 & 2.7 & 3.0 & 0.0 & \\
\hline NSD1 & c.589A>C & p.T197P & COSM3341014 & 3.0 & 1.5 & 0.0 & $\begin{array}{c}\text { Neutral } \\
\text { (score 0.20) }\end{array}$ \\
\hline NSD1 & c.946T>G & p.S316A & COSM4384048 & 1.0 & 0.0 & 3.3 & \\
\hline RNF213 & c.12305G>A & p.R826* & COSM5641817 & 0.0 & 1.7 & 1.4 & $\begin{array}{c}\text { Neutral } \\
\text { (score 0.02) }\end{array}$ \\
\hline
\end{tabular}

Fig. 6 Patient specific features of tumor suppressor gene mutation 
somatic mutation in esophageal squamous cell carcinoma (ESCC), medulloblastoma, follicular lymphoma, and diffuse large B-cell lymphoma patients [28]. More intriguingly, G. Paolo et al. showed that the frequency of $K M T 2 C / D$ gene mutation was also notable in patients with histology of Cutaneous squamous cell carcinoma (SCC), head and neck SCC, lung SCC, esophageal SCC, and cervical SCC [29]. In similar to these studies, our results indicate that mutation rates of ZFHX3, KMT2C, KMT2D, NSD1, and RNF213 genes have existed at a high frequency in CC patients in despite of the characteristics of these genetic mutations that have not been clearly identified in CC patients. The reason is that $79 \%$ of histologic subtype of $\mathrm{CC}$ in our cohort is consisted of squamous cell carcinoma (Table 1). Therefore, our findings on these genetic variations may be applicable to future studies of the molecular mechanism of cervical cancer.

In addition, RNF213 mutation, which was employed as a monitoring marker for CC patients in our study, is currently little known about its function and role in cancer. RNF213 is primarily known as E3 ubiquitin-protein ligase involved in angiogenesis and non-canonical signal pathway in vascular development. A preceding study is mainly focused on Moyamoya disease, and a cerebrovascular disease characterized by progressive bilateral stenosis of internal carotid arteries [30]. Therefore, the role of RNF213 in cervical cancer is required for further investigation and must be validated as a prognostic factor to measure clinical outcomes during cervical cancer treatment.

We report several important aspects regarding the promising application of cfDNA for early diagnosis and monitoring of CC: (i) Gene mutation can serve as a prognostic biomarker for detecting $\mathrm{CC}$ by the profiling of the tumor suppressor and cancer driver genes. (ii) Mutations in tumor suppressor genes are prevalent in all stages of CC, and (iii) Chemotherapy and radiotherapy affect the allele frequency, which can be utilized for monitoring cancer. We also report the comprehensive mutation profile of CC samples. Notably, frequently mutated genes, such as TP53 or PIK3CA in CC, were not predominantly identified in 24 Korean women. Interestingly, during the course of treatment of $\mathrm{CC}$, we discovered that continuous observation of Tumor suppressor gene mutations could be employed to reveal the appropriate treatment modalities in patients. This approach of using liquid biopsy to detect the mutation pattern can be used in clinical practice. Although specific anticancer drugs for CC treatment have not yet been approved by the Food and Drug Administration, drugs prescribed for other carcinomas or radiation therapy can be used. Additionally, NGS technology, which can be explicitly used for the diagnosis of $\mathrm{CC}$, needs to have a more accurate clinical specificity by minimizing false-positive diagnosis.

\section{Conclusion}

$\mathrm{CC}$ is the cause of malignancy-related death among women. For the clinically usage in clinicians and patients parts, we developed the NGS CC panel. Through NGS analysis with blood samples in Korean women, the genetic variations in $\mathrm{CC}$ were found that are related to the genetic alteration result of TCGA and COSMIC data. Although we have some technical tasks to improve, we showed the advanced step of CC diagnosis with the NGS technology with blood. Our multifaceted approach to assessing genetic variations can be used for the diagnosis, monitoring, and further treatment of CC.

\section{Supplementary information}

Supplementary information accompanies this paper at https://doi.org/10. 1186/s12885-020-07161-0.

Additional file 1: Supplementary Table 1. The list of genetic variants at baseline.

\section{Abbreviations}

AF: Allele Frequency; CC: Cervical Cancer; cfDNA: Cell-free DNA; ctDNA: Circulating tumor DNA; CR: Complete Response;

FDG: Fluordeoxyglucose; HR-HPV: High-risk Human papillomavirus; ICR: Intracavity Radiation; LN: Lymph node; NGS: Next-GenerationSequencing; PBMC: Peripheral Blood Mononuclear Cell; PR: Partial Response; SCC: Squamous cell carcinoma; SNP: Single Nucleotide Polymorphism; TCGA: The Cancer Genome Atlas

\section{Acknowledgments}

We thank to the participants who made this study possible. We thank A.B. and YAC. for the open discussion and manuscript editing.

\section{Authors' contributions}

SYL and DKC were major contributors in conceptualization, design, data collection, analysis, and wrote the manuscript. YHL, JB and DB wrote the manuscript and interpreted data regarding NGS data. JHA and $\mathrm{CHC}$ ware involved data collection and conducted the experiments. DHC interpreted the data regarding cervical cancer and NGS data and supervised the experiments and analyses. SHL and BCK participated in improving the manuscript and curated data. All authors finally approved the version to be published and agree to be accountable for all aspects of the work.

\section{Funding}

This study was partly supported by grant of the Basic Research Program (2017R1A2B4012353) and the Bio \& Medical Technology Development Program (2017M3A9F7074175) of the Nation Research Foundation (NRF) funded by the Ministry of Science \& ICT, Republic of Korea. This study was also supported by the Reserch Base Construction Fund Support Program funded by Jeonbuk National University in 2019. This study was part of the Investment-linked Corporate Growth R\&D Support Program (KOl-

TAR\&D150405) by Korea Industrial Technology Association. JB was supported by the Genome Korea Project in Ulsan Research Fund (1.180024.01 and

1.180017.01) of UNIST. This paper was also supported by Fund of Biomedical Research Institute, Jeonbuk National University Hospital.

\section{Availability of data and materials}

Not applicable.

\section{Ethics approval and consent to participate}

Administrative permission was acquired to access data used in the research. All patients provided written informed consent for study participation, and all clinical specimens were collected with approval from the institutional review board (IRB No. CUH2017-04-018-001) and ethics committee of Jeonbuk National University Hospital. 


\section{Consent for publication}

Not applicable.

\section{Competing interests}

The authors declare that they have no competing interests.

\section{Author details}

'Department of Radiation Oncology, Jeonbuk National University Hospital-Jeonbuk National University Medical School, Jeonju, Jeonbuk, Republic of Korea. ${ }^{2}$ Research Institute of Clinical Medicine of Jeonbuk National University-Biomedical Research Institute of Jeonbuk National University Hospital, Jeonju, Republic of Korea. ${ }^{3}$ Clinomics Inc, Suwon 16229, Republic of Korea. ${ }^{4}$ Department of Biophysics and Radiation Biology, Lab of Nanochemistry, Semmelweis University, Budapest, Hungary. ${ }^{5} \mathrm{KOGIC}$, UNIST Ulsan 44919, Republic of Korea. ${ }^{6}$ Geromics LTD, Cambridge CB1 1AH, UK. ${ }^{7}$ Department of Obstetrics and Gynecology, Jeonbuk National University Hospital-Jeonbuk National University Medical School, Jeonju, Jeonbuk, Republic of Korea.

Received: 11 December 2019 Accepted: 9 July 2020

Published online: 27 July 2020

\section{References}

1. Allemani C, et al. Global surveillance of cancer survival 1995-2009: analysis of individual data for 25,676,887 patients from 279 population-based registries in 67 countries (CONCORD-2). Lancet (London, England). 2015;385: 977-1010.

2. Pogoda CS, Roden RB, Garcea RL. Immunizing against Anogenital Cancer: HPV vaccines. PLoS Pathog. 2016:12:e1005587.

3. Woodman CB, Collins SI, Young LS. The natural history of cervical HPV infection: unresolved issues. Nat Rev Cancer. 2007;7:11-22.

4. Roden RBS, Stern PL. Opportunities and challenges for human papillomavirus vaccination in cancer. Nat Rev Cancer. 2018;18:240-54.

5. Elit L, Fyles AW, Oliver TK, Devries-Aboud MC, Fung-Kee-Fung M. Follow-up for women after treatment for cervical cancer. Curr Oncol (Toronto, Ont). 2010;17:65-9.

6. Volik S, Alcaide M, Morin RD, Collins C. Cell-free DNA (cfDNA): clinical significance and utility in Cancer shaped by emerging technologies. Mo Cancer Res. 2016;14:898-908.

7. Salvi $\mathrm{S}$, et al. Cell-free DNA as a diagnostic marker for cancer: current insights. Onco Targets Ther. 2016;9:6549-59.

8. The Lancet, O. Liquid cancer biopsy: the future of cancer detection? Lancet Oncol. 2016;17:123.

9. Kang Z, Stevanovic S, Hinrichs CS, Cao L. Circulating cell-free DNA for metastatic cervical Cancer detection, genotyping, and monitoring. Clin Cancer Res. 2017;23:6856-62.

10. Cheung TH, et al. Liquid biopsy of HPV DNA in cervical cancer. J Clin Virol. 2019;114:32-6.

11. Tian J, et al. Using plasma cell free DNA to monitor the chemo-radiotherapy course of cervical cancer. Int J Cancer. 2019;145:2547-57.

12. Kim J, et al. KoVariome: Korean National Standard Reference Variome database of whole genomes with comprehensive SNV, indel, CNV, and SV analyses. Sci Rep. 2018:8:5677.

13. Cho YS, et al. An ethnically relevant consensus Korean reference genome is a step towards personal reference genomes. Nat Commun. 2016;7:13637.

14. Cancer Genome Atlas Research, N, et al. Integrated genomic and molecular characterization of cervical cancer. Nature. 2017:543:378-84

15. Sappino AP, et al. The CEACAM1 tumor suppressor is an ATM and p53regulated gene required for the induction of cellular senescence by DNA damage. Oncogenesis. 2012;1:e7.

16. De La Rosa-Velazquez IA, Rincon-Arano H, Benitez-Bribiesca L, Recillas-Targa F. Epigenetic regulation of the human retinoblastoma tumor suppressor gene promoter by CTCF. Cancer Res. 2007;67:2577-85.

17. Walker CJ, et al. Patterns of CTCF and ZFHX3 Mutation and Associated Outcomes in Endometrial Cancer. J Natl Cancer Inst. 2015;107:djv249.

18. Gudbjartsson DF, et al. A sequence variant in ZFHX3 on 16q22 associates with atrial fibrillation and ischemic stroke. Nat Genet. 2009;41:876-8.

19. Hu Q, et al. ZFHX3 is indispensable for ERbeta to inhibit cell proliferation via MYC downregulation in prostate cancer cells. Oncogenesis. 2019;8:28.
20. Dawkins JB, et al. Reduced expression of histone Methyltransferases KMT2C and KMT2D correlates with improved outcome in pancreatic ductal adenocarcinoma. Cancer Res. 2016;76:4861-71.

21. Rabello DDA, et al. MLL2/KMT2D and MLL3/KMT2C expression correlates with disease progression and response to imatinib mesylate in chronic myeloid leukemia. Cancer Cell Int. 2018;18:26.

22. Malan V, et al. Sotos syndrome caused by a paracentric inversion disrupting the NSD1 gene. Clin Genet. 2008;73:89-91.

23. Su X, et al. NSD1 inactivation and SETD2 mutation drive a convergence toward loss of function of $\mathrm{H} 3 \mathrm{~K} 36$ writers in clear cell renal cell carcinomas. Cancer Res. 2017;77:4835-45.

24. Kaikkonen MU, Spann NJ, Heinz S, et al. Remodeling of the enhancer landscape during macrophage activation is coupled to enhancer transcription. Mol Cell. 2013;51:310-25.

25. Wang C, et al. Enhancer priming by H3K4 methyltransferase MLL4 controls cell fate transition. Proc Natl Acad Sci U S A. 2016;113:11871-6.

26. Dorighi KM, et al. MII3 and MII4 facilitate enhancer RNA synthesis and transcription from promoters independently of H3K4 Monomethylation. Mol Cell. 2017:66:568-76.

27. Rickels R, et al. Histone H3K4 monomethylation catalyzed by Trr and mammalian COMPASS-like proteins at enhancers is dispensable for development and viability. Nat Genet. 2017:49:1647-53.

28. Gao YB, et al. Genetic landscape of esophageal squamous cell carcinoma. Nat Genet. 2014;46:1097-110.

29. Dotto GP, Rustgi AK. Squamous cell cancers: a unified perspective on biology and genetics. Cancer Cell. 2016:29:622-37.

30. Smith ER, Scott RM. Spontaneous occlusion of the circle of Willis in children: pediatric moyamoya summary with proposed evidence-based practice guidelines. A review. J Neurosurg Pediatr. 2012;9:353-60.

\section{Publisher's Note}

Springer Nature remains neutral with regard to jurisdictional claims in published maps and institutional affiliations.
Ready to submit your research? Choose BMC and benefit from:

- fast, convenient online submission

- thorough peer review by experienced researchers in your field

- rapid publication on acceptance

- support for research data, including large and complex data types

- gold Open Access which fosters wider collaboration and increased citations

- maximum visibility for your research: over $100 \mathrm{M}$ website views per year

At BMC, research is always in progress.

Learn more biomedcentral.com/submissions 